\title{
On the Binding Energy Parameter $\lambda$ of Common Envelope Evolution
}

\author{
Xiao-Jie $\mathrm{Xu}^{1,2}$ and Xiang-Dong $\mathrm{Li}^{1,2}$ \\ ${ }^{1}$ Department of Astronomy, Nanjing University, Nanjing 210093, China; \\ ${ }^{2}$ Key laboratory of Modern Astronomy and Astrophysics (Nanjing University), Ministry of \\ Education, Nanjing 210093, China \\ lixd@nju.edu.cn
}

Received __; accepted _ 


\begin{abstract}
The binding energy parameter $\lambda$ plays an important role in common envelope (CE) evolution. Previous works have already pointed out that $\lambda$ varies throughout the stellar evolution, though it has been adopted as a constant in most of the population synthesis calculations. We have systematically calculated the binding energy parameter $\lambda$ for both Population I and Population II stars of masses $1-20 M_{\odot}$, taking into account the contribution from the internal energy of stellar matter. We present fitting formulae for $\lambda$ that can be incorporated into future population synthesis investigations. We also briefly discuss the possible applications of the results in binary evolutions.
\end{abstract}

Subject headings: stars: evolution - stars: mass loss - binaries: general 


\section{Introduction}

One of the key stages in the evolution of close binary stars is the common envelope (CE) evolution. When the primary star fills its Roche-lobe (RL) and the following mass transfer is dynamically unstable so that the secondary cannot accrete all the transferred material, the transferred matter will form an envelope embedding both stars and the binary enters the CE evolution. The secondary star orbits inside the CE, and experiences a drag force by the envelope. This causes the orbital decay and spiral-in of the star with possible ejection of the envelope (for reviews see Iben \& Livio 1993; Taam \& Sandquist 2000). Two main kinds of theories have been developed to describe the envelope ejection process. One is the $\alpha$ formalism, which considers the conversion of orbital energy to overcome the envelope binding energy (Webbink 1984; de Kool 1990; Dewi \& Tauris 2000). If the orbital energy of the secondary is large enough to eject the envelope, the system will survive and become a compact binary containing the core of the lobe-filling star and the secondary in a much smaller orbit; if the orbital energy is not enough, the two stars will coalesce. The other is the $\gamma$ formalism, which considers angular momentum transformation during the spiral-in (see Nelemans \& Tout 2005, and reference therein). Despite extensive three dimensional hydrodynamical simulations of $\mathrm{CE}$ evolution (for recent reviews, see Taam \& Sandquist 2000; Taam \& Ricker 2006), the physics of the CE phase remains poorly understood, including the efficiency with which the CE is ejected from the system. Alternative evolutionary paths have been proposed to avoid the CE evolution, such as those by King \& Begelman (1999) and Beer et al. (2007), who suggested that super-Eddington accretion may lead to mass ejection from the system instead of entering the CE stage.

According to Han et al. (1994, 1995) and Dewi \& Tauris (2000), the total binding energy of the envelope is described by:

$$
E_{\mathrm{bind}}=\int_{M_{\mathrm{core}}}^{M_{\mathrm{donor}}}-\frac{G M(r)}{r} \mathrm{~d} m+\alpha_{\mathrm{th}} \int_{M_{\text {core }}}^{M_{\mathrm{donor}}} U \mathrm{~d} m,
$$


where $M_{\text {donor }}$ is the mass of the donor star and $M_{\text {core }}$ is its core mass, $-G M(r) / r$ and $U$ are the gravitational and internal energy of the stellar matter respectively, $G$ is the gravitational constant, and $\alpha_{\text {th }}$ the percentage of the internal energy contributing to ejection of the envelope, normally taking the value between 0 and 1 .

For convenience the total binding energy of the envelope is usually expressed as (Webbink 1984; de Kool 1990; Dewi \& Tauris 2000):

$$
E_{\text {bind }}=-\frac{G M_{\text {donor }} M_{\text {env }}}{\lambda a_{\mathrm{i}} r_{\mathrm{L}}},
$$

where $M_{\text {env }}$ is the envelope mass, $\lambda$ is the binding energy parameter, $r_{\mathrm{L}}=R_{\mathrm{L}} / a_{\mathrm{i}}$ is the ratio of the RL radius and the orbital separation at the onset of $\mathrm{CE}$, and $a_{\mathrm{i}} r_{\mathrm{L}}$ is normally taken as the stellar radius once a star fills its RL and starts to transfer matter. If we assume that part of the change of the orbital energy is used to eject the envelope, as described by Webbink (1984), then

$$
E_{\text {bind }}=\alpha_{\mathrm{CE}}\left(\frac{G M_{\text {core }} M_{2}}{2 a_{\mathrm{f}}}-\frac{G M_{\mathrm{donor}} M_{2}}{2 a_{\mathrm{i}}}\right)
$$

where $\alpha_{\mathrm{CE}}$ is the efficiency of converting the orbital energy to kinetic energy to eject the CE, $M_{2}$ the mass of the secondary, and $a_{\mathrm{i}}$ and $a_{\mathrm{f}}$ refer to the initial and final orbital separation of the CE phase, respectively.

Combine Eqs. (2) and (3), the final orbital separation is related to the pre-CE separation by the following formula,

$$
\frac{a_{\mathrm{f}}}{a_{\mathrm{i}}}=\frac{M_{\text {core }} M_{2}}{M_{\text {donor }}} \frac{1}{M_{2}+2 M_{\text {env }} / \alpha_{\mathrm{CE}} \lambda r_{\mathrm{L}}} .
$$

Equation (4) indicates that the orbital separation after CE is sensitively dependent on the product of $\alpha_{\mathrm{CE}}$ and $\lambda$, and the convenient way is to treat both $\alpha_{\mathrm{CE}}$ and $\lambda$ as constants of the order of unity. In most of the population synthesis investigations concerning the evolution of close binaries in the literature, $\lambda$ was usually taken to be a constant, normally around 0.5 
throughout the evolution of the system. However, there have lots of works (e.g. Han et al. 1994; Dewi \& Tauris 2000, 2001; Podsiadlowski et al.|2003; Webbink 2007) suggesting that $\lambda$ is a variable. Dewi \& Tauris (2000) and Dewi \& Tauris (2001) found that the value of $\lambda$ changes as the star evolves, and reaches far more than 0.5 in late evolutionary stages for stars with mass between 3 and $6 M_{\odot}$. In recent population synthesis on post-CE binaries, Davis et al. (2010) used linear interpolations of the tabular data in Dewi \& Tauris (2000) to calculate $\lambda$. We notice that Dewi \& Tauris (2000) and Dewi \& Tauris (2001)'s calculations did not cover Pop. I stars less massive than $3 M_{\odot}$ and Pop. II stars, and their results in tabular form may not be easily used. Obviously more advanced population synthesis requires a simple description of the parameter $\lambda$ for better constraints on the initial stellar population. In this work we are attempting to investigate the binding energy parameter $\lambda$ by considering the contribution of internal energy of stellar matter for both Pop. I and Pop. II stars, and to find easy-to-use fitting formulae of $\lambda$ for further research.

This paper is organized as follows. We describe the stellar evolution code and assumptions adopted in Sect. 2. In Sect. 3 we present the calculated results. We summarize our results and briefly discuss their possible implications in Sect. 4.

\section{Model Description}

We adopt the latest stellar evolution code TWIN $\sqrt{1}$ developed by Eggleton (1971) and modified later by Eggleton (1972), Eggleton et al. (1973) and Pols et al. (1995). One of

\footnotetext{
${ }^{1}$ The code used here is a variant of the code $e v$ described, in its initial version, by Eggleton (1971, 1972) and Eggleton et al. (1973), and was developed by the present authors from the version of Eggleton (2009, unpublished). The current version of ev is obtainable on request from eggleton1@llnl.gov, along with data tables and a user manual.
} 
the major improvements of the code is that, when a low-mass star is reaching the He flash igniting point, the code will automatically generate a zero-age horizontal branch star with both the same core and total mass which enables further evolution.

We take the mixing length parameter $\alpha=l / H_{p}$ to be 2.0 and follow the evolution of both Pop. I and Pop. II stars. We assume the chemical composition of $X=0.7$ and $Z=0.02$ for Pop. I stars and $X=0.755$ and $Z=0.001$ for Pop. II ones. Once a star evolves off the main-sequence, we use the layer that contains less than $15 \% \mathrm{H}$ as the boundary between the core and the envelope. We then calculate the envelope binding energy from Eq. (11) by setting $\alpha_{\text {th }}$ to be either 0 or 1 . After that we use Eq. (21) to calculate the corresponding value of $\lambda$, where we simply take $a_{\mathrm{i}} r_{\mathrm{L}}=R_{\mathrm{L}}$ as the stellar radius $R$. To include the stellar wind mass loss during the evolution, we consider the following mechanisms similar to Hurley et al. (2000).

1. The modified Nieuwenhuijzen-type wind model (Nieuwenhuijzen \& de Jager 1990):

$$
\dot{M}_{\mathrm{NJ}}=9.6 \times 10^{-15}\left(\frac{Z}{Z_{\odot}}\right)^{1 / 2} R^{0.81} L^{1.24} M^{0.16} M_{\odot} \mathrm{yr}^{-1}
$$

where $M, R$, and $L$ are the stellar mass, radius and luminosity in solar units, respectively. The wind mass loss rate is modified by the factor $Z^{1 / 2}$ due to different metallicity (Kudritzki et al. 1987). The influence of metallicity on the strengths of stellar winds arises from the fact that the effective number of lines contributing to the line acceleration changes with metallicity $Z$ and, hence, causes a metallicity dependence of the mass-loss rate. The observations of Puls et al. (1996), which in the metallicity range between the Galaxy and the $\operatorname{SMC}\left(Z \sim Z_{\odot} / 5\right)$ confirms the exponent of $1 / 2$ for O-stars.

2. The Reimers type wind for giant-branch stars as described by Kudritzki \& Reimers (1978) and Iben \& Renzini (1983):

$$
\dot{M}_{\mathrm{R}}=4 \times 10^{-13} \frac{\eta L R}{M} M_{\odot \mathrm{yr}^{-1}}
$$


with $\eta=0.5$ to modify the wind loss.

3. The reduced Wolf-Rayet-like mass loss by Hamann. Koesterke \& Wessolowski (1995) and Hamann \& Koesterke (1998):

$$
\dot{M}_{\mathrm{WR}}=10^{-13} L^{1.5}(1.0-\mu) M_{\odot} \mathrm{yr}^{-1},
$$

with

$$
\mu=\left(\frac{M-M_{\text {core }}}{M}\right) \min \left\{5.0, \max \left[1.2,\left(\frac{L}{L_{0}}\right)^{\kappa}\right],\right\}
$$

where $L_{0}=7.0 \times 10^{4}$ and $\kappa=-0.5$.

4. AGB stellar wind loss by Vassiliadis \& Wood (1993):

$$
\log \dot{M}_{\mathrm{VW}}=-11.4+0.0125\left[P_{0}-100 \max (M-2.5,0.0)\right] M_{\odot} \mathrm{yr}^{-1},
$$

where

$$
\log P_{0}=\min (3.3,-2.07-0.9 \log M+1.94 \log R),
$$

and this wind loss is limited to

$$
\dot{M}_{\mathrm{VW}, \max }=1.36 \times 10^{-9} L M_{\odot} \mathrm{yr}^{-1} .
$$

Then the total mass loss via stellar wind (absolute value) is taken to be

$$
\dot{M}=\max \left(\dot{M}_{\mathrm{NJ}}, \dot{M}_{\mathrm{R}}, \dot{M}_{\mathrm{WR}}, \dot{M}_{\mathrm{VW}}\right) .
$$

When taking into account the contribution of internal energy to total binding energy, we include the following terms: the thermal energy of the stellar matter, the radiation energy, the ionization of $\mathrm{H}, \mathrm{H}^{+}, \mathrm{He}, \mathrm{He}^{+}$and $\mathrm{He}^{++}$, the dissociation of $\mathrm{H}_{2}$, the ionization of C, N, O, Ne, Mg, Si and Fe (these seven elements are assumed to be fully ionized for all the temperatures). To descriminate the effect of internal energy, we set $\alpha_{\text {th }}$ to be 0 and 1 , and calculate the respective binding energy parameter, namely $\lambda_{\mathrm{g}}$ and $\lambda_{\mathrm{b}}$, respectively. 


\section{Calculation Results}

We have made calculations for both Pop. I and Pop. II stars, with mass range from 1 to $20 M_{\odot}$. For each star we follow its evolution until its age exceeds 15 Gyr or the code crushes for rapid C burning. Once a star evolves to produce a H-exhausted core we calculate the binding energy of its envelope and accordingly $\lambda_{\mathrm{b}}$ and $\lambda_{\mathrm{g}}$, both with and without the internal energy of the stellar matter. In Figs. 1 and 2 we show some examples of the evolution of $\lambda_{\mathrm{b}}$ and $\lambda_{\mathrm{g}}$ with respect to the stellar radius $R$.

Since a star experiences both expansion and shrinkage throughout its life, we divide its evolution into three stages after the star leaves the main-sequence according to the change of the stellar radius, to make the fitting of $\lambda$ more practicable. Stage 1 begins at the center $\mathrm{H}$ exhaustion and ends when the star starts to shrink (i.e., near center He ignition). Stage 2 follows and ends when the star starts to expand again 2 . Stage 3 begins after that and continues till the end of the evolution.

Figures 1 and 2 show that both $\lambda_{\mathrm{b}}$ and $\lambda_{\mathrm{g}}$ vary during the evolution of stars. For $1 M_{\odot}$ star, both $\lambda_{\mathrm{b}}$ and $\lambda_{\mathrm{g}}$ decrease with $R$, but their magnitudes are around unity before the He flash. After that the values of $\lambda$ have a big decrease along with the stellar radius, but $\lambda_{\mathrm{b}} \sim 2 \lambda_{\mathrm{g}}$ throughout the evolution. More massive stars often experience rapid increase in $\lambda$ in stage 3 . For stars with mass from $\sim 3$ to $5 M_{\odot}$ (the mass range is related to

\footnotetext{
${ }^{2}$ Generally a star shrinks after its center He ignition and starts to expand again later. For convenience of fitting we define the end point of stage 2 (and also the starting point of stage 3) as the minimum of stellar radius. For example, for Pop I stars which are more massive than $10 M_{\odot}$, we use $10^{-7}$ center He abundance as the end point of stage 2 . As a less massive star expands again before its center He exhaustion, we simply assume that stage 2 ends when the respective HB star starts to expand again.
} 
metallicity), $\lambda_{\mathrm{b}}$ and $\lambda_{\mathrm{g}}$ take the values $\sim 1$ before the star reaches stage 3 , during which the internal energy dominates, and the total binding energy finally becomes positive. The resulting $\lambda_{\mathrm{b}}$ increases rapidly and eventually turns out to be infinity, while $\lambda_{\mathrm{g}}$ remains to be around 0.5 . For stars massive than $6 M_{\odot}$, the binding energy decreases in stage 3 but never becomes positive, correspondingly $\lambda_{\mathrm{b}}$ takes smaller value and $\lambda_{\mathrm{g}}$ is around 0.5. For stars more massive than $12 M_{\odot}, \lambda$ even drops below 0.5 for most $R$. The extreme case in our calculation is for $20 M_{\odot}$ stars, in which both $\lambda_{\mathrm{b}}$ and $\lambda_{\mathrm{g}}$ evolve to be $\ll 1$ in stage 3 .

To provide easy-to-use formulae of $\lambda_{\mathrm{b}}$ and $\lambda_{\mathrm{g}}$, we make polynomial fitting with the following formulation,

$$
y=a+b_{1} x+b_{2} x^{2}+b_{3} x^{3}+b_{4} x^{4}+b_{5} x^{5},
$$

where $y$ is $\lambda_{\mathrm{b}}$ or $\lambda_{\mathrm{g}}$, and $x$ is the stellar radius $R$ in most cases. For low-mass stars, we also use the fractional mass of the envelope $m_{\mathrm{env}}=M_{\mathrm{env}} / M_{\mathrm{donor}}$ as the fitting variable as

suggested by Webbink (2007). In limited cases we use $\log \lambda_{\mathrm{b}}$ instead of $\lambda_{\mathrm{b}}$ as the $y$ variable during stage 3 when the value of $\lambda_{\mathrm{b}}$ is too large, and take $1 / \lambda$ as $y$ and $m_{\mathrm{env}}$ as $x$ in several cases (see Tables 1 and 2 for details). In Tables 1 and 2 we present the fitting parameters for Pop. I and Pop. II stars, respectively.

\section{Discussion and conclusions}

Our calculations show that stars with different masses have different values of $\lambda$, and $\lambda$ is not constant for the same star in different evolutionary stages. From Figs. 1] and 2, $\lambda_{\mathrm{b}}$ diverges from 0.5 for most $M$ and $R$, and it is obvious that assuming a constant $\lambda=0.5$ in population synthesis calculations is far from fact and therefore lack of reliability. It is also interesting to note that the range of $\lambda_{\mathrm{g}}$ is much narrower than that of $\lambda_{\mathrm{b}}$. Of course the actual value of $\lambda$ should lie between $\lambda_{\mathrm{g}}$ and $\lambda_{\mathrm{b}}$ since not all of the internal energy 
contributes to the ejection of the envelope (Dewi \& Tauris 2000). As we have given the fitting formulae for $\lambda$, the results can be useful for future population synthesis works. However, this approach should be adopted carefully, especially in the cases when the binding energies are positive 3 , and where the efficiency of conversion of ionization energy to kinetic energy may differ from the efficiency of conversion of other forms of internal energy. As we can see from Eqs. (11) and (2), the calculated values of $\lambda$ depend on the stellar mass, the core mass, the envelope mass distribution and the percentage of internal energy that contributes to envelope ejection. Factors like metallicity and stellar wind mass loss also affect the stellar evolution and the resulting values of $\lambda$. Additionally, the different definitions of core-envelope boundary lead to different stellar core mass and therefore affect the calculated results.

Our results agree reasonably with that of Han et al. (1994), Dewi \& Tauris (2000) and Dewi \& Tauris (2001), although there exist some differences. Dewi \& Tauris (2000) proposed that $\lambda$ is almost independent of the stellar chemical composition. From our calculations we find that given the same mass, Pop. I and Pop. II stars have different $\lambda$-values (see Figs. 1 and 21). For example, the mass range in which the values of $\lambda_{\mathrm{b}}$ can become negative is narrower for Pop. II stars than for Pop. I ones. In Fig. 3 we compare the evolution of the two $\lambda_{\mathrm{s}}$ for a $5 M_{\odot}$ star with different metallicity. It is seen that the $\lambda$ evolutions show little difference at the beginning, but later they diverge from each other, and the values of $\lambda$ for the Pop. I star always lie above those of the Pop. II star in late

\footnotetext{
${ }^{3}$ Although the gravitational binding energy remains negative for all the stars throughout their evolution, the total binding energy, on the other hand, can turn to be positive for stars with mass ranging from $\sim 3-5 M_{\odot}$ (specifically, 3 to $5.6 M_{\odot}$ for Pop. I stars and 2.8 to $4.5 M_{\odot}$ for Pop. II stars at the end of their AGB phase when their radii expand to be more than hundreds of solar radius, and ionizaiton energy dominates the total binding energy).
} 
evolutionary stages. The reason is as follows. The binding energy (absolute value) of the Pop. I star is either comparable with (in early evolutionary stage) or lower than (due to the contribution of ionization energy) that of the Pop. II star. Since the Pop. I star evolves slower than the Pop. II one, as a result the Pop. I star has a smaller H-exhausted core and higher-mass envelope than the Pop. II star when they have the same radii, thus higher $\lambda$ values.

We used the $15 \% \mathrm{H}$ abundance layer as the stellar core-envelope boundary to calculate the binding energy and $\lambda$, since the core mass from such definition increases in most of the time and is suitable for calculating the binding energy (Eggleton 2009). Note that Dewi \& Tauris (2000) used the $10 \% \mathrm{H}$ abundance layer as the core-envelope boundary, and in our calculation we found that the two definitions give similar results. There are other criteria to define this boundary, such as the maximum energy generation layer or the density gradient criteria (Han et al. 1994; Dewi \& Tauris 2000). According to Tauris \& Dewi (2001) and Dewi \& Tauris (2001), most of these criteria give outer core boundary and higher core mass. In general, if the total stellar mass is a constant, a more massive core will decrease the total binding energy of the envelope and result in a larger $\lambda$-value (Dewi \& Tauris 2000; Tauris \& Dewi 2001; Dewi \& Tauris 2001). In other words, the $\lambda$-values we calculated may be regarded as the lower limit of the actual ones. Stars more massive than $10 M_{\odot}$ lose $10 \%$ to $30 \%$ of its total mass during its evolution, which can affect the stellar structure and the values of $\lambda$ (Dewi \& Tauris 2000, 2001; Podsiadlowski et al. 2003). Dewi \& Tauris (2001) and Podsiadlowski et al. (2003) have also found that $\lambda$ is sensitive to the wind mass loss for massive stars, and including stellar wind leads to higher binding energy (absolute value) and lower value of $\lambda$.

Our results suggest that $\lambda_{\mathrm{b}}$ can take values significantly larger than 0.5 during the late-stage evolution of stars with mass between $\sim 2$ and $10 M_{\odot}$, which will significantly 
increase the efficiency of envelope ejection. If we consider an evolved giant or AGB star in this mass range with $\lambda>>0.5$, the envelope binding energy may be much smaller compared to that with $\lambda=0.5$. It is then much easier for its companion to eject the CE, and to produce wider binaries after the CE phase. It is also worth noticing that both $\lambda_{\mathrm{g}}$ and $\lambda_{\mathrm{b}}$ are less than 1 in the late evolutionary stages of massive stars. For stars massive than $\sim 8 M_{\odot}, \lambda$ even lies below 0.2. As a result, the actual envelope ejection efficiency for these stars decreases considerably. Taking a $20 M_{\odot}$ Pop. I giant star as an example. As seen from Fig. 1, both $\lambda_{\mathrm{b}}$ and $\lambda_{\mathrm{g}}$ are $\ll 1$, which means that the envelope binding energy become much higher. The secondary has to convert more orbital energy to eject the envelope, which probably leads to a much tighter final orbit, or even a merger. These may have important implications for the formation and evolution of related binaries that produce compact stars, which will be discussed in future works in more detail.

We thank Prof. P. P. Eggleton for providing the latest version of the evolutionary code and helpful suggestions. This work was supported by the Natural Science Foundation of China (under grant number 10873008) and the National Basic Research Program of China (973 Program 2009CB824800). 


\section{REFERENCES}

Beer, M. E., Dray, L. M., King, A. R., \& Wynn, G. A. 2007, MNRAS, 375, 1000

Davis, P. J., Kolb, U., \& Willems, B. 2010, MNRAS, 403, 179

de Kool, M. 1990, ApJ, 358, 189

Dewi, J., \& Tauris, T. 2000, A\&A, 360, 1043

Dewi, J., \& Tauris, T. 2001, ASP Conf., 229, 255

Eggleton, P. P., 1971, MNRAS, 151, 351

Eggleton, P. P., 1972, MNRAS, 156, 361

Eggleton, P. P., Faulkner, J., \& Flannery, B. P. 1973, A\&A, 23, 325

Hamann, W. R., Koesterke, L., \& Wessolowski, U. 1995, A\&A, 299, 151

Hamann, W. R., \& Koesterke, L. 1998, A\&A, 335, 1003

Han, Z., Podsiadlowski, P., \& Eggleton, P. P. 1994, MNRAS, 271, 121

Han, Z., Podsiadlowski, P., \& Eggleton, P. P. 1995, MNRAS, 272, 800

Hurley, J. R., Pols, O. R., \& Tou, C. A. 2000, MNRAS, 315, 543

Iben, I. Jr, \& Renzini, A. 1993, PASP, 105, 1373

Iben, I. Jr, \& Renzini, A. 1983, ARA\&A, 21, 271

King, A. R., \& Begelman M. C. 1999, ApJ, 519, L169

Kudritzki, R. P., \& Reimers, D. 1978, A\&A, 70, 227

Kudritzki, R. P., Pauldrach, A., \& Puls, J. 1987, A\&A, 173, 293 
Nelemans, G., \& Tout, C. A. 2005, MNRAS, 356, 753

Nieuwenhuijzen, H., \& de Jager, C. 1990, A\&A, 231, 134

Podsiadlowski, P., Rappaport, S., \& Han, Z. 2003, MNRAS, 341, 385

Pols, O. R., Tout, C. A., Eggleton, P. P, \& Han, Z. 1995, MNRAS, 274, 964

Puls, J., Kudritzki, R. P., Herrero, A., Pauldrach., A. W. A., Haser, S. M., et al. 1996, A\&A, 307, 171

Taam, R. E., \& Sandquist E. L. 2000, ARA\&A, 38, 113

Taam, R. E., \& Ricker, P. M. 2006, to appear in proceedings of "A life with stars", a conference in honor of Ed van den Heuvel's 60th birthday (astro-ph/0611043)

Tauris, T., \& Dewi, J. 2001, A\&A, 369, 170

Vassiliadis, E., \& Wood, P. R. 1993, ApJ, 413, 641

Webbink, R. F. 1984, ApJ, 277, 355

Webbink, R. F. 2007, in Short Period Binary Stars, ed. E.F. Milone, D.A. Leahy, \& D.W. Hobill (Berlin: Springer), 233 (arXiv:0704.0280) 

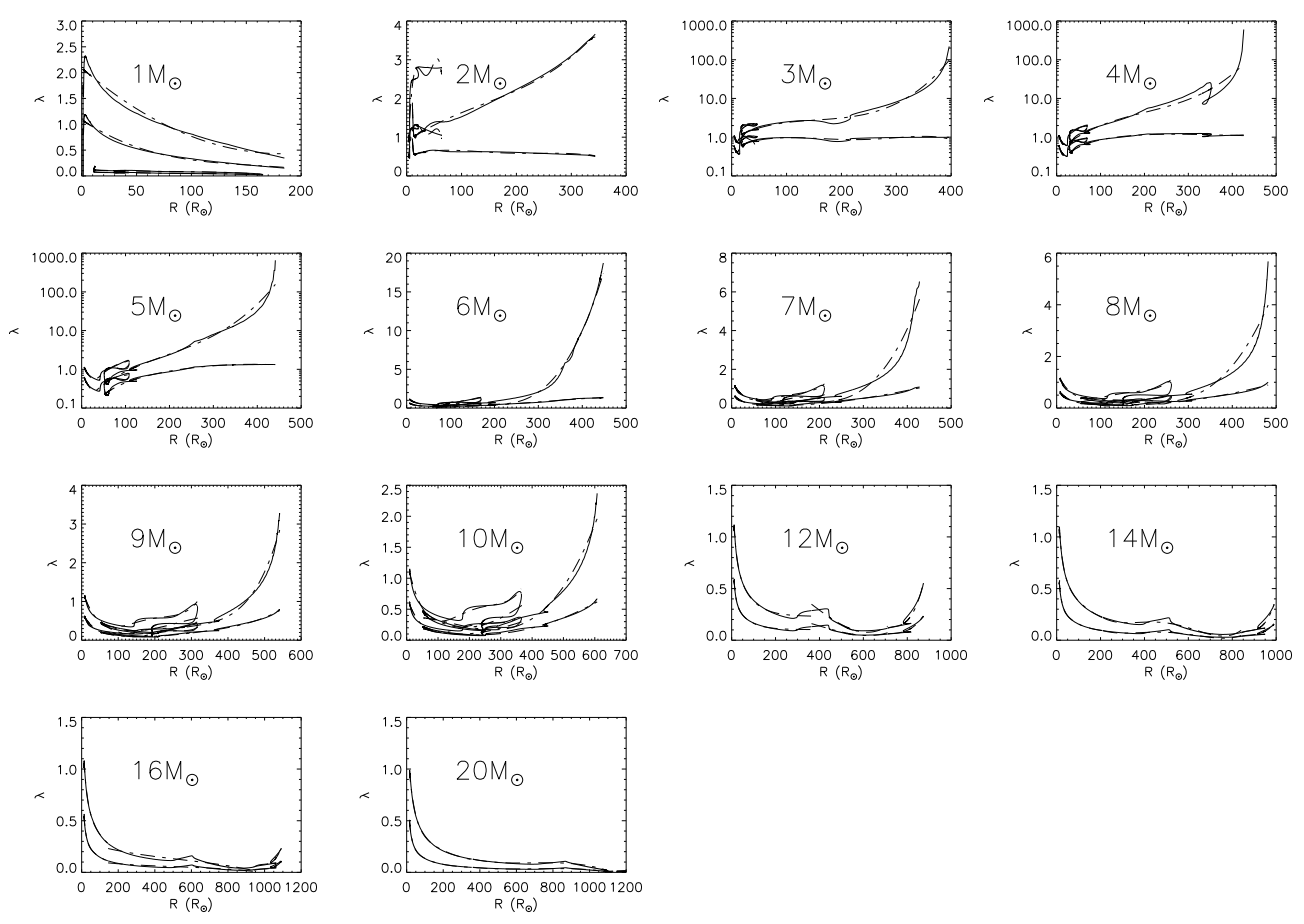

Fig. 1. - The evolution of the binding energy parameter $\lambda$ for Pop. I stars of mass $1 M_{\odot}$ to $20 M_{\odot}$. The upper and lower solid lines are $\lambda_{\mathrm{b}}$ and $\lambda_{\mathrm{g}}$, respectively. The dot-dashed lines are the fitting results. 

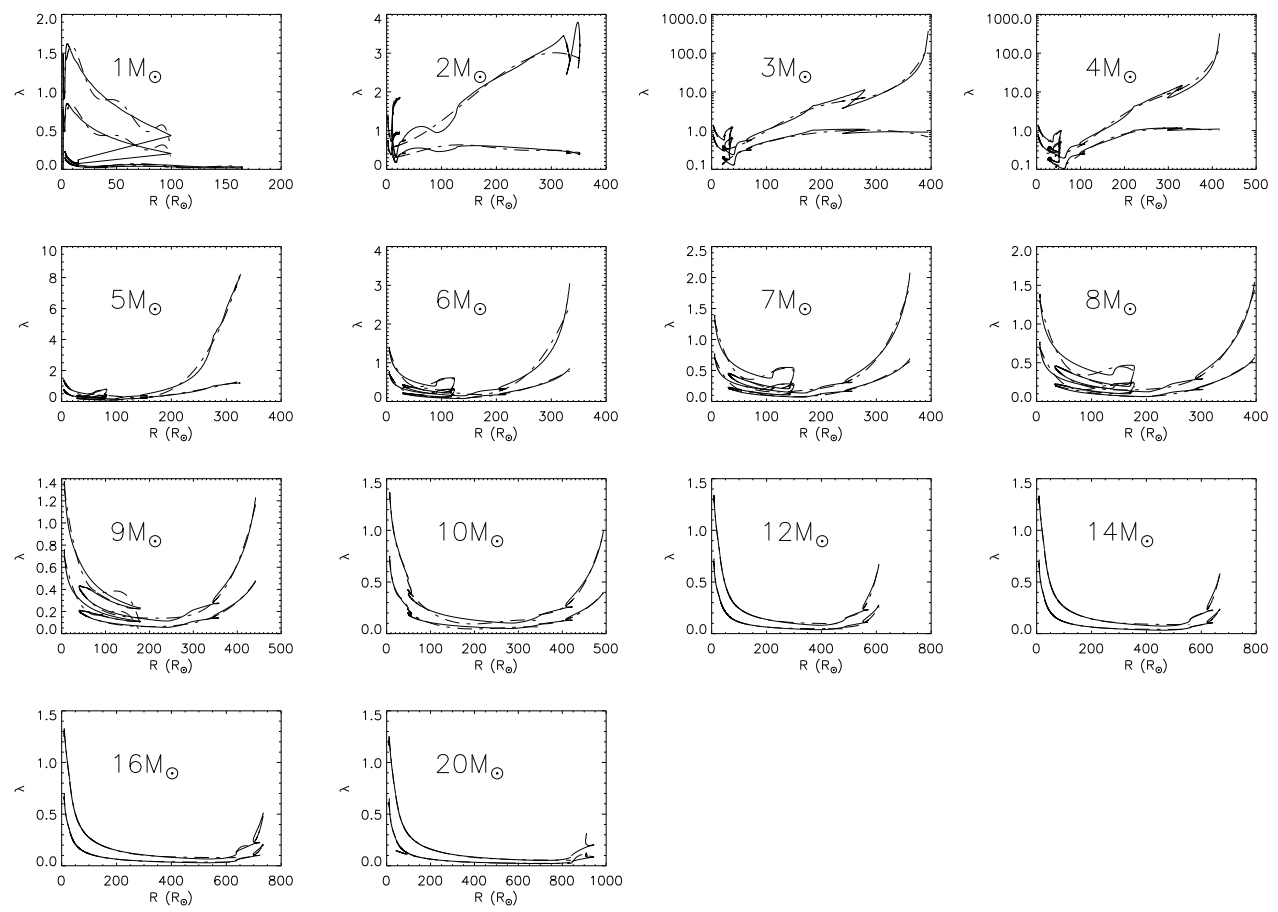

Fig. 2.- Same as Fig. 1 but for Pop. II stars. 


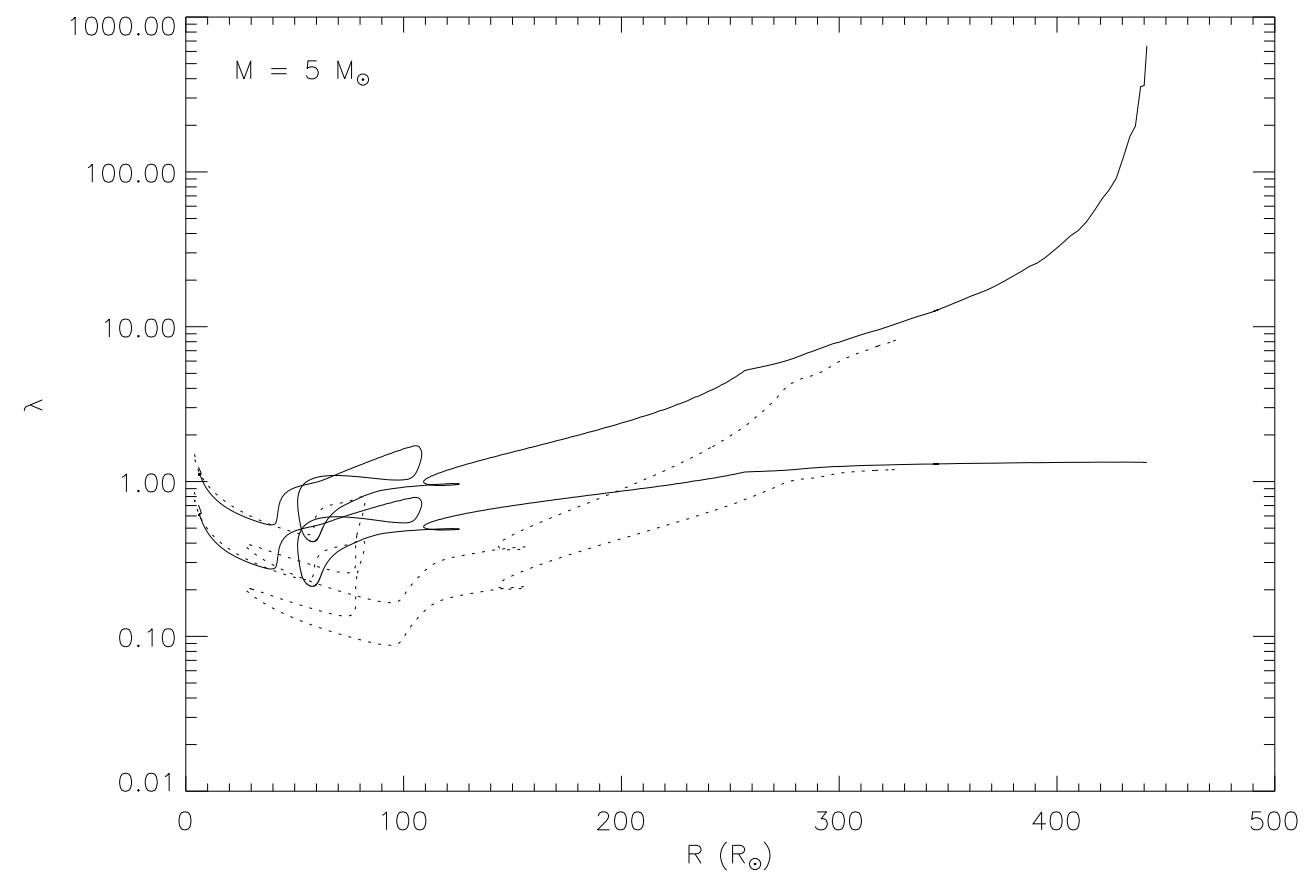

Fig. 3.- Comparison of the $\lambda$ values for $5 M_{\odot}$ Pop. I and Pop. II stars. The upper and lower solid lines represent $\lambda_{\mathrm{b}}$ and $\lambda_{\mathrm{g}}$ for the Pop. I star, respectively. The upper and lower dotted lines are $\lambda_{\mathrm{b}}$ and $\lambda_{\mathrm{g}}$ for the Pop. II star, respectively. 
Table 1. Fitting Results of $\lambda$ for Pop. I Stars

\begin{tabular}{|c|c|c|c|c|c|c|c|c|}
\hline Stage & $\operatorname{Mass}\left(M_{\odot}\right)$ & $\lambda$ & $\mathrm{a}$ & $b_{1}$ & $b_{2}$ & $b_{3}$ & $b_{4}$ & $b_{5}$ \\
\hline \multirow[t]{2}{*}{1} & $1^{* *}$ & $\lambda_{b}$ & 19.84809 & -69.00242 & 81.24502 & -31.5572 & 0 & 0 \\
\hline & $* *$ & $\lambda_{g}$ & 26.09283 & -72.37166 & 64.13808 & -16.24247 & 0 & 0 \\
\hline \multirow[t]{2}{*}{1} & 2 & $\lambda_{b}$ & 2.05363 & -0.00685 & $-3.42739 \mathrm{E}-4$ & $3.93987 \mathrm{E}-6$ & $-1.18237 \mathrm{E}-8$ & 0 \\
\hline & & $\lambda_{g}$ & 1.07658 & -0.01041 & $-4.90553 \mathrm{E}-5$ & $1.13528 \mathrm{E}-6$ & $-3.91609 \mathrm{E}-9$ & 0 \\
\hline \multirow[t]{2}{*}{1} & 3 & $\lambda_{b}$ & 2.40831 & -0.42459 & 0.03431 & $-9.26879 \mathrm{E}-4$ & $8.24522 \mathrm{E}-6$ & 0 \\
\hline & & $\lambda_{g}$ & 1.30705 & -0.22924 & 0.01847 & $-5.06216 \mathrm{E}-4$ & $4.57098 \mathrm{E}-6$ & 0 \\
\hline \multirow[t]{2}{*}{1} & 4 & $\lambda_{b}$ & 1.8186 & -0.17464 & 0.00828 & $-1.31727 \mathrm{E}-4$ & $7.08329 \mathrm{E}-7$ & 0 \\
\hline & & $\lambda_{g}$ & 1.02183 & -0.1024 & 0.00493 & $-8.16343 \mathrm{E}-5$ & $4.55426 \mathrm{E}-7$ & 0 \\
\hline \multirow[t]{2}{*}{1} & 5 & $\lambda_{b}$ & 1.52581 & -0.08125 & 0.00219 & $-2.0527 \mathrm{E}-5$ & $6.79169 \mathrm{E}-8$ & 0 \\
\hline & & $\lambda_{g}$ & 0.85723 & -0.04922 & 0.00137 & $-1.36163 \mathrm{E}-5$ & $4.68683 \mathrm{E}-8$ & 0 \\
\hline \multirow[t]{2}{*}{1} & 6 & $\lambda_{b}$ & 1.41601 & -0.04965 & $8.51527 \mathrm{E}-4$ & $-5.54384 \mathrm{E}-6$ & $1.32336 \mathrm{E}-8$ & 0 \\
\hline & & $\lambda_{g}$ & 0.78428 & -0.02959 & $5.2013 \mathrm{E}-4$ & $-3.45172 \mathrm{E}-6$ & $8.17248 \mathrm{E}-9$ & 0 \\
\hline \multirow[t]{2}{*}{1} & 7 & $\lambda_{b}$ & 1.38344 & -0.04093 & $5.78952 \mathrm{E}-4$ & $-3.19227 \mathrm{E}-6$ & $6.40902 \mathrm{E}-9$ & 0 \\
\hline & & $\lambda_{g}$ & 0.76009 & -0.02412 & $3.47104 \mathrm{E}-4$ & $-1.92347 \mathrm{E}-6$ & 3.79609E-9 & 0 \\
\hline \multirow[t]{2}{*}{1} & 8 & $\lambda_{b}$ & 1.35516 & -0.03414 & $4.02065 \mathrm{E}-4$ & $-1.85931 \mathrm{E}-6$ & $3.08832 \mathrm{E}-9$ & 0 \\
\hline & & $\lambda_{g}$ & 0.73826 & -0.01995 & $2.37842 \mathrm{E}-4$ & $-1.09803 \mathrm{E}-6$ & $1.79044 \mathrm{E}-9$ & 0 \\
\hline \multirow[t]{2}{*}{1} & 9 & $\lambda_{b}$ & 1.32549 & -0.02845 & $2.79097 \mathrm{E}-4$ & $-1.07254 \mathrm{E}-6$ & $1.46801 \mathrm{E}-9$ & 0 \\
\hline & & $\lambda_{g}$ & 0.71571 & -0.01657 & $1.64607 \mathrm{E}-4$ & $-6.31935 \mathrm{E}-7$ & $8.52082 \mathrm{E}-10$ & 0 \\
\hline \multirow[t]{2}{*}{1} & 10 & $\lambda_{b}$ & 1.29312 & -0.02371 & $1.93764 \mathrm{E}-4$ & $-6.19576 \mathrm{E}-7$ & $7.04227 \mathrm{E}-10$ & 0 \\
\hline & & $\lambda_{g}$ & 0.69245 & -0.01398 & $1.17256 \mathrm{E}-4$ & $-3.81487 \mathrm{E}-7$ & $4.35818 \mathrm{E}-10$ & 0 \\
\hline \multirow[t]{2}{*}{1} & 12 & $\lambda_{b}$ & 0.23031 & 0.74955 & 13.20774 & 0.63332 & 68.67896 & 0 \\
\hline & & $\lambda_{g}$ & 0.10247 & 0.31709 & 60.57985 & 0.7254 & 8.90151 & 0 \\
\hline \multirow[t]{2}{*}{1} & 14 & $\lambda_{b}$ & 0.0576 & 0.59416 & 158.80613 & 0.87013 & 19.04369 & 0 \\
\hline & & $\lambda_{g}$ & 0.07178 & 0.29286 & 80.4363 & 0.73414 & 10.52307 & 0 \\
\hline \multirow[t]{2}{*}{1} & 16 & $\lambda_{b}$ & -0.2064 & 0.88945 & 22.23505 & 0.79813 & 287.58284 & 0 \\
\hline & & $\lambda_{g}$ & 0.04116 & 0.74011 & 11.60388 & 0.29533 & 97.68433 & 0 \\
\hline \multirow[t]{2}{*}{1} & 20 & $\lambda_{b}$ & 0.04959 & 0.85626 & 29.90918 & 0.44041 & 199.20324 & 0 \\
\hline & & $\lambda_{g}$ & 0.04101 & 0.24106 & 105.06077 & 0.68656 & 14.81172 & 0 \\
\hline \multirow[t]{2}{*}{2} & $1^{* *}$ & $\lambda_{b}$ & 16.71634 & -26.38217 & -14.18029 & 0 & 0 & 0 \\
\hline & $* *$ & $\lambda_{g}$ & 24.27819 & -31.47029 & -33.30049 & 0 & 0 & 0 \\
\hline
\end{tabular}


Table 1-Continued

\begin{tabular}{|c|c|c|c|c|c|c|c|c|}
\hline Stage & Mass $\left(M_{\odot}\right)$ & $\lambda$ & $\mathrm{a}$ & $b_{1}$ & $b_{2}$ & $b_{3}$ & $b_{4}$ & $b_{5}$ \\
\hline \multirow[t]{2}{*}{2} & 2 & $\lambda_{b}$ & 34.41826 & -6.65259 & 0.43823 & -0.00953 & 0 & 0 \\
\hline & & $\lambda_{g}$ & 13.66058 & -2.48031 & 0.15275 & -0.00303 & 0 & 0 \\
\hline \multirow[t]{2}{*}{2} & 3 & $\lambda_{b}$ & -42.98513 & 7.90134 & -0.54646 & 0.01863 & $-3.13101 \mathrm{E}-4$ & $2.07468 \mathrm{E}-6$ \\
\hline & & $\lambda_{g}$ & -6.73842 & 1.06656 & -0.05344 & 0.00116 & $-9.34446 \mathrm{E}-6$ & 0 \\
\hline \multirow[t]{2}{*}{2} & 4 & $\lambda_{b}$ & -7.3098 & 0.56647 & -0.01176 & $7.90112 \mathrm{E}-5$ & 0 & 0 \\
\hline & & $\lambda_{g}$ & -3.80455 & 0.29308 & -0.00603 & $4.00471 \mathrm{E}-5$ & 0 & 0 \\
\hline \multirow[t]{2}{*}{2} & 5 & $\lambda_{b}$ & -9.93647 & 0.42831 & -0.00544 & $2.25848 \mathrm{E}-5$ & 0 & 0 \\
\hline & & $\lambda_{g}$ & -5.33279 & 0.22728 & -0.00285 & $1.16408 \mathrm{E}-5$ & 0 & 0 \\
\hline \multirow[t]{2}{*}{2} & 6 & $\lambda_{b}$ & 13.91465 & -0.55579 & 0.00809 & $-4.94872 \mathrm{E}-5$ & $1.08899 \mathrm{E}-7$ & 0 \\
\hline & & $\lambda_{g}$ & 7.68768 & -0.30723 & 0.00445 & $-2.70449 \mathrm{E}-5$ & $5.89712 \mathrm{E}-8$ & 0 \\
\hline \multirow[t]{2}{*}{2} & 7 & $\lambda_{b}$ & 4.12387 & -0.12979 & 0.00153 & $-7.43227 \mathrm{E}-6$ & $1.29418 \mathrm{E}-8$ & 0 \\
\hline & & $\lambda_{g}$ & 2.18952 & -0.06892 & $8.00936 \mathrm{E}-4$ & $-3.78092 \mathrm{E}-6$ & $6.3482 \mathrm{E}-9$ & 0 \\
\hline \multirow[t]{2}{*}{2} & 8 & $\lambda_{b}$ & -3.89189 & 0.19378 & -0.0032 & $2.39504 \mathrm{E}-5$ & $-8.28959 \mathrm{E}-8$ & $1.07843 \mathrm{E}-10$ \\
\hline & & $\lambda_{g}$ & -2.24354 & 0.10918 & -0.00179 & $1.33244 \mathrm{E}-5$ & $-4.57829 \mathrm{E}-8$ & 5.90313E-11 \\
\hline \multirow[t]{2}{*}{2} & 9 & $\lambda_{b}$ & 0.86369 & -0.00995 & $4.80837 \mathrm{E}-5$ & $-6.10454 \mathrm{E}-8$ & $-2.79504 \mathrm{E}-12$ & 0 \\
\hline & & $\lambda_{g}$ & -0.7299 & 0.0391 & $-5.78132 \mathrm{E}-4$ & $3.7072 \mathrm{E}-6$ & $-1.07036 \mathrm{E}-8$ & $1.14833 \mathrm{E}-11$ \\
\hline \multirow[t]{2}{*}{2} & 10 & $\lambda_{b}$ & 0.74233 & -0.00623 & $2.04197 \mathrm{E}-5$ & $-1.30388 \mathrm{E}-8$ & 0 & 0 \\
\hline & & $\lambda_{g}$ & 0.36742 & -0.00344 & $1.27838 \mathrm{E}-5$ & $-1.0722 \mathrm{E}-8$ & 0 & 0 \\
\hline \multirow[t]{2}{*}{2} & $12^{* *}$ & $\lambda_{b}$ & -7261.84589 & 43423.48906 & -96937.03708 & 95946.58045 & -35560.07607 & 0 \\
\hline & $* *$ & $\lambda_{g}$ & -280.84015 & 1259.61941 & -1674.61224 & 681.20633 & 0 & 0 \\
\hline \multirow[t]{2}{*}{2} & $14^{* *}$ & $\lambda_{b}$ & -3334.04459 & 20638.88263 & -47238.6357 & 47684.78906 & -17966.67435 & 0 \\
\hline & $* *$ & $\lambda_{g}$ & -4769.9922 & 29195.29936 & -65789.75807 & 65234.39892 & -24107.00589 & 0 \\
\hline \multirow[t]{2}{*}{2} & $16^{* *}$ & $\lambda_{b}$ & -321.34016 & 1789.56304 & -3002.4542 & 1600.03159 & 0 & 0 \\
\hline & $* *$ & $\lambda_{g}$ & -683.28449 & 3792.87458 & -6376.86784 & 3419.38525 & 0 & 0 \\
\hline \multirow[t]{2}{*}{2} & $20^{* *}$ & $\lambda_{b}$ & 72.31855 & 482.85039 & -2593.27601 & 3880.69092 & -1913.27392 & 0 \\
\hline & $* *$ & $\lambda_{g}$ & 166.95028 & 649.50464 & -3805.22907 & 5447.28964 & -2487.34665 & 0 \\
\hline \multirow[t]{2}{*}{3} & $1^{* *}$ & $\lambda_{b}$ & 75.23035 & -763.8397 & 3180.89498 & -4665.23406 & 0 & 0 \\
\hline & $* *$ & $\lambda_{g}$ & 121.25389 & -1169.84175 & 4686.7113 & -6749.8215 & 0 & 0 \\
\hline \multirow[t]{2}{*}{3} & 2 & $\lambda_{b}$ & 0.88954 & 0.0098 & $-3.1411 \mathrm{E}-5$ & 7.66979E-8 & 0 & 0 \\
\hline & & $\lambda_{g}$ & 0.48271 & 0.00584 & $-6.22051 \mathrm{E}-5$ & $2.41531 \mathrm{E}-7$ & $-3.1872 \mathrm{E}-10$ & 0 \\
\hline
\end{tabular}


Table 1-Continued

\begin{tabular}{|c|c|c|c|c|c|c|c|c|}
\hline Stage & $\operatorname{Mass}\left(M_{\odot}\right)$ & $\lambda$ & $\mathrm{a}$ & $b_{1}$ & $b_{2}$ & $b_{3}$ & $b_{4}$ & $b_{5}$ \\
\hline \multirow[t]{2}{*}{3} & $3^{*}$ & $\lambda_{b}$ & -0.04669 & 0.00764 & $-4.32726 \mathrm{E}-5$ & $9.31942 \mathrm{E}-8$ & 0 & 0 \\
\hline & & $\lambda_{g}$ & 0.44889 & 0.01102 & $-6.46629 \mathrm{E}-5$ & $5.66857 \mathrm{E}-9$ & $7.21818 \mathrm{E}-10$ & $-1.2201 \mathrm{E}-12$ \\
\hline \multirow[t]{2}{*}{3} & $4^{*}$ & $\lambda_{b}$ & -0.37322 & 0.00943 & $-3.26033 \mathrm{E}-5$ & $5.37823 \mathrm{E}-8$ & 0 & 0 \\
\hline & & $\lambda_{g}$ & 0.13153 & 0.00984 & $-2.89832 \mathrm{E}-5$ & $2.63519 \mathrm{E}-8$ & 0 & 0 \\
\hline \multirow[t]{2}{*}{3} & $5^{*}$ & $\lambda_{b}$ & -0.80011 & 0.00992 & $-3.03247 \mathrm{E}-5$ & $5.26235 \mathrm{E}-8$ & 0 & 0 \\
\hline & & $\lambda_{g}$ & -0.00456 & 0.00426 & 4.71117E-6 & $-1.72858 \mathrm{E}-8$ & 0 & 0 \\
\hline \multirow[t]{2}{*}{3} & 6 & $\lambda_{b}$ & -2.7714 & 0.06467 & $-4.01537 \mathrm{E}-4$ & $7.98466 \mathrm{E}-7$ & 0 & 0 \\
\hline & & $\lambda_{g}$ & 0.23083 & -0.00266 & $2.21788 \mathrm{E}-5$ & $-2.35696 \mathrm{E}-8$ & 0 & 0 \\
\hline \multirow[t]{2}{*}{3} & 7 & $\lambda_{b}$ & -0.63266 & 0.02054 & $-1.3646 \mathrm{E}-4$ & $2.8661 \mathrm{E}-7$ & 0 & 0 \\
\hline & & $\lambda_{g}$ & 0.26294 & -0.00253 & $1.32272 \mathrm{E}-5$ & $-7.12205 \mathrm{E}-9$ & 0 & 0 \\
\hline \multirow[t]{2}{*}{3} & 8 & $\lambda_{b}$ & -0.1288 & 0.0099 & $-6.71455 \mathrm{E}-5$ & $1.33568 \mathrm{E}-7$ & 0 & 0 \\
\hline & & $\lambda_{g}$ & 0.26956 & -0.00219 & 7.97743E-6 & $-1.53296 \mathrm{E}-9$ & 0 & 0 \\
\hline \multirow[t]{2}{*}{3} & 9 & $\lambda_{b}$ & 1.19804 & -0.01961 & $1.28222 \mathrm{E}-4$ & $-3.41278 \mathrm{E}-7$ & $3.35614 \mathrm{E}-10$ & 0 \\
\hline & & $\lambda_{g}$ & 0.40587 & -0.0051 & $2.73866 \mathrm{E}-5$ & $-5.74476 \mathrm{E}-8$ & $4.90218 \mathrm{E}-11$ & 0 \\
\hline \multirow[t]{2}{*}{3} & 10 & $\lambda_{b}$ & 0.3707 & $2.67221 \mathrm{E}-4$ & $-9.86464 \mathrm{E}-6$ & $2.26185 \mathrm{E}-8$ & 0 & 0 \\
\hline & & $\lambda_{g}$ & 0.25549 & -0.00152 & 3.35239E-6 & $2.24224 \mathrm{E}-10$ & 0 & 0 \\
\hline \multirow[t]{2}{*}{3} & 12 & $\lambda_{b}$ & -58.03732 & 0.23633 & $-3.20535 \mathrm{E}-4$ & $1.45129 \mathrm{E}-7$ & 0 & 0 \\
\hline & & $\lambda_{g}$ & -15.11672 & 0.06331 & $-8.81542 \mathrm{E}-5$ & $4.0982 \mathrm{E}-8$ & 0 & 0 \\
\hline \multirow[t]{2}{*}{3} & 14 & $\lambda_{b}$ & -106.90553 & 0.36469 & $-4.1472 \mathrm{E}-4$ & $1.57349 \mathrm{E}-7$ & 0 & 0 \\
\hline & & $\lambda_{g}$ & -39.93089 & 0.13667 & $-1.55958 \mathrm{E}-4$ & $5.94076 \mathrm{E}-8$ & 0 & 0 \\
\hline \multirow[t]{2}{*}{3} & 16 & $\lambda_{b}$ & -154.70559 & 0.46718 & $-4.70169 \mathrm{E}-4$ & $1.57773 \mathrm{E}-7$ & 0 & 0 \\
\hline & & $\lambda_{g}$ & -65.39602 & 0.19763 & $-1.99078 \mathrm{E}-4$ & $6.68766 \mathrm{E}-8$ & 0 & 0 \\
\hline \multirow[t]{2}{*}{3} & $20^{* *}$ & $\lambda_{b}$ & -260484.85724 & $4.26759 \mathrm{E} 6$ & $-2.33016 \mathrm{E} 7$ & $4.24102 \mathrm{E} 7$ & 0 & 0 \\
\hline & $* *$ & $\lambda_{g}$ & -480055.67991 & $7.87484 \mathrm{E} 6$ & $-4.30546 \mathrm{E} 7$ & $7.84699 \mathrm{E} 7$ & 0 & 0 \\
\hline
\end{tabular}

* Rows with the symbol $*$ uses $\log \lambda_{b}$ instead of $\lambda_{b}$ as $y$ variables.

** Rows with the symbol ** use $m_{\mathrm{env}}=M_{\mathrm{env}} / M$ instead of $R$ as $x$ variables and $1 / \lambda$ as $y$ variables. 
Table 2. Fitting Results of $\lambda$ for Pop. II Stars

\begin{tabular}{|c|c|c|c|c|c|c|c|c|}
\hline Stage & $\operatorname{Mass}\left(M_{\odot}\right)$ & $\lambda$ & $\mathrm{a}$ & $b_{1}$ & $b_{2}$ & $b_{3}$ & $b_{4}$ & $b_{5}$ \\
\hline \multirow[t]{2}{*}{1} & 1 & $\lambda_{b}$ & 1.06031 & 0.08173 & -0.00436 & $6.7847 \mathrm{E}-5$ & $-3.3429 \mathrm{E}-7$ & 0 \\
\hline & & $\lambda_{g}$ & 0.57192 & 0.03937 & -0.00221 & $3.49192 \mathrm{E}-5$ & $-1.73046 \mathrm{E}-7$ & 0 \\
\hline \multirow[t]{2}{*}{1} & 2 & $\lambda_{b}$ & 2.56108 & -0.75562 & 0.1027 & -0.00495 & $8.05436 \mathrm{E}-5$ & 0 \\
\hline & & $\lambda_{g}$ & 1.41896 & -0.4266 & 0.05792 & -0.00281 & $4.61 \mathrm{E}-5$ & 0 \\
\hline \multirow[t]{2}{*}{1} & 3 & $\lambda_{b}$ & 1.7814 & -0.17138 & 0.00754 & $-9.02652 \mathrm{E}-5$ & 0 & 0 \\
\hline & & $\lambda_{g}$ & 0.99218 & -0.10082 & 0.00451 & $-5.53632 \mathrm{E}-5$ & 0 & 0 \\
\hline \multirow[t]{2}{*}{1} & 4 & $\lambda_{b}$ & 1.65914 & -0.10398 & 0.0029 & $-2.24862 \mathrm{E}-5$ & 0 & 0 \\
\hline & & $\lambda_{b}$ & 0.92172 & -0.06187 & 0.00177 & $-1.42677 \mathrm{E}-5$ & 0 & 0 \\
\hline \multirow[t]{2}{*}{1} & 5 & $\lambda_{b}$ & 1.58701 & -0.06897 & 0.00129 & $-6.99399 \mathrm{E}-6$ & 0 & 0 \\
\hline & & $\lambda_{g}$ & 0.87647 & -0.04103 & 7.91444E-4 & $-4.41644 \mathrm{E}-6$ & 0 & 0 \\
\hline \multirow[t]{2}{*}{1} & 6 & $\lambda_{b}$ & 1.527 & -0.04738 & $6.1373 \mathrm{E}-4$ & $-2.36835 \mathrm{E}-6$ & 0 & 0 \\
\hline & & $\lambda_{g}$ & 0.83636 & -0.02806 & $3.73346 \mathrm{E}-4$ & $-1.47016 \mathrm{E}-6$ & 0 & 0 \\
\hline \multirow[t]{2}{*}{1} & 7 & $\lambda_{b}$ & 1.49995 & -0.03921 & $4.2327 \mathrm{E}-4$ & $-1.37646 \mathrm{E}-6$ & 0 & 0 \\
\hline & & $\lambda_{g}$ & 0.81688 & -0.02324 & $2.5804 \mathrm{E}-4$ & $-8.54696 \mathrm{E}-7$ & 0 & 0 \\
\hline \multirow[t]{2}{*}{1} & 8 & $\lambda_{b}$ & 1.46826 & -0.03184 & $2.85622 \mathrm{E}-4$ & $-7.91228 \mathrm{E}-7$ & 0 & 0 \\
\hline & & $\lambda_{g}$ & 0.79396 & -0.01903 & $1.77574 \mathrm{E}-4$ & $-5.04262 \mathrm{E}-7$ & 0 & 0 \\
\hline \multirow[t]{2}{*}{1} & 9 & $\lambda_{b}$ & 1.49196 & -0.03247 & $3.08066 \mathrm{E}-4$ & $-9.53247 \mathrm{E}-7$ & 0 & 0 \\
\hline & & $\lambda_{g}$ & 0.805 & -0.02 & $2.01872 \mathrm{E}-4$ & $-6.4295 \mathrm{E}-7$ & 0 & 0 \\
\hline \multirow[t]{2}{*}{1} & 10 & $\lambda_{b}$ & 1.73966 & -0.07074 & 0.00178 & $-1.73072 \mathrm{E}-5$ & 0 & 0 \\
\hline & & $\lambda_{g}$ & 1.027 & -0.05685 & 0.00164 & $-1.65206 \mathrm{E}-5$ & 0 & 0 \\
\hline \multirow[t]{2}{*}{1} & 12 & $\lambda_{b}$ & 1.63634 & -0.04646 & 7.49351E-4 & $-5.23622 \mathrm{E}-6$ & 0 & 0 \\
\hline & & $\lambda_{g}$ & 1.17934 & -0.08481 & 0.00329 & $-4.69096 \mathrm{E}-5$ & 0 & 0 \\
\hline \multirow[t]{2}{*}{1} & 14 & $\lambda_{b}$ & 1.45573 & -0.00937 & -0.00131 & $3.07004 \mathrm{E}-5$ & 0 & 0 \\
\hline & & $\lambda_{g}$ & 1.19526 & -0.08503 & 0.00324 & $-4.58919 \mathrm{E}-5$ & 0 & 0 \\
\hline \multirow[t]{2}{*}{1} & 16 & $\lambda_{b}$ & 1.33378 & 0.01274 & -0.00234 & $4.6036 \mathrm{E}-5$ & 0 & 0 \\
\hline & & $\lambda_{g}$ & 1.17731 & -0.07834 & 0.00275 & $-3.58108 \mathrm{E}-5$ & 0 & 0 \\
\hline \multirow[t]{2}{*}{1} & 20 & $\lambda_{b}$ & 1.27138 & 0.00538 & -0.0012 & $1.80776 \mathrm{E}-5$ & 0 & 0 \\
\hline & & $\lambda_{g}$ & 1.07496 & -0.05737 & 0.00153 & $-1.49005 \mathrm{E}-5$ & 0 & 0 \\
\hline \multirow[t]{2}{*}{2} & 1 & $\lambda_{b}$ & 0.37294 & -0.05825 & 0.00375 & $-7.59191 \mathrm{E}-5$ & 0 & 0 \\
\hline & & $\lambda_{g}$ & 0.24816 & -0.04102 & 0.0028 & $-6.20419 \mathrm{E}-5$ & 0 & 0 \\
\hline
\end{tabular}


Table 2-Continued

\begin{tabular}{|c|c|c|c|c|c|c|c|c|}
\hline Stage & $\operatorname{Mass}\left(M_{\odot}\right)$ & $\lambda$ & $\mathrm{a}$ & $b_{1}$ & $b_{2}$ & $b_{3}$ & $b_{4}$ & $b_{5}$ \\
\hline \multirow[t]{2}{*}{2} & 2 & $\lambda_{b}$ & -103.92538 & 25.37325 & -2.03273 & 0.0543 & 0 & 0 \\
\hline & & $\lambda_{g}$ & -56.03478 & 13.6749 & -1.09533 & 0.02925 & 0 & 0 \\
\hline \multirow[t]{2}{*}{2} & 3 & $\lambda_{b}$ & -12.40832 & 1.59021 & -0.06494 & $8.69587 \mathrm{E}-4$ & 0 & 0 \\
\hline & & $\lambda_{g}$ & -6.47476 & 0.8328 & -0.03412 & $4.58399 \mathrm{E}-4$ & 0 & 0 \\
\hline \multirow[t]{2}{*}{2} & 4 & $\lambda_{b}$ & -5.89253 & 0.54296 & -0.01527 & $1.38354 \mathrm{E}-4$ & 0 & 0 \\
\hline & & $\lambda_{g}$ & -3.21299 & 0.29583 & -0.00833 & $7.55646 \mathrm{E}-5$ & 0 & 0 \\
\hline \multirow[t]{2}{*}{2} & 5 & $\lambda_{b}$ & -0.67176 & 0.07708 & -0.00175 & $1.1991 \mathrm{E}-5$ & 0 & 0 \\
\hline & & $\lambda_{g}$ & -0.38561 & 0.0427 & $-9.6948 \mathrm{E}-4$ & $6.64455 \mathrm{E}-6$ & 0 & 0 \\
\hline \multirow[t]{2}{*}{2} & 6 & $\lambda_{b}$ & 0.30941 & 0.00965 & $-2.31975 \mathrm{E}-4$ & $1.26273 \mathrm{E}-6$ & 0 & 0 \\
\hline & & $\lambda_{g}$ & 0.14576 & 0.00562 & $-1.30273 \mathrm{E}-4$ & $7.06459 \mathrm{E}-7$ & 0 & 0 \\
\hline \multirow[t]{2}{*}{2} & 7 & $\lambda_{b}$ & 0.44862 & 0.00234 & $-9.23152 \mathrm{E}-5$ & $4.67797 \mathrm{E}-7$ & 0 & 0 \\
\hline & & $\lambda_{g}$ & 0.21873 & 0.00154 & $-5.18806 \mathrm{E}-5$ & $2.60283 \mathrm{E}-7$ & 0 & 0 \\
\hline \multirow[t]{2}{*}{2} & 8 & $\lambda_{b}$ & 0.50221 & $-3.19021 \mathrm{E}-4$ & $-3.81717 \mathrm{E}-5$ & $1.80726 \mathrm{E}-7$ & 0 & 0 \\
\hline & & $\lambda_{g}$ & 0.24748 & $-9.9338 \mathrm{E}-5$ & $-1.99272 \mathrm{E}-5$ & $9.47504 \mathrm{E}-8$ & 0 & 0 \\
\hline \multirow[t]{2}{*}{2} & 9 & $\lambda_{b}$ & 0.39342 & 0.00259 & $-4.97778 \mathrm{E}-5$ & $1.69533 \mathrm{E}-7$ & 0 & 0 \\
\hline & & $\lambda_{g}$ & 0.20796 & $6.62921 \mathrm{E}-4$ & $-1.84663 \mathrm{E}-5$ & $6.58983 \mathrm{E}-8$ & 0 & 0 \\
\hline \multirow[t]{2}{*}{2} & 10 & $\lambda_{b}$ & 0.75746 & -0.00852 & $3.51646 \mathrm{E}-5$ & $-4.57725 \mathrm{E}-8$ & 0 & 0 \\
\hline & & $\lambda_{g}$ & 0.35355 & -0.00388 & $1.56573 \mathrm{E}-5$ & $-1.98173 \mathrm{E}-8$ & 0 & 0 \\
\hline \multirow[t]{2}{*}{2} & 12 & $\lambda_{b}$ & 0.09472 & 1.59771 & 19.38009 & 0.4662 & 83.08091 & 0 \\
\hline & & $\lambda_{g}$ & 0.04621 & 0.23145 & 76.88504 & 0.64758 & 18.45577 & 0 \\
\hline \multirow[t]{2}{*}{2} & 14 & $\lambda_{b}$ & 0.08576 & 0.50546 & 90.52062 & 1.73793 & 17.7433 & 0 \\
\hline & & $\lambda_{g}$ & 0.04015 & 0.69617 & 17.24357 & 0.23011 & 86.67988 & 0 \\
\hline \multirow[t]{2}{*}{2} & 16 & $\lambda_{b}$ & 0.0774 & 1.86228 & 17.55952 & 0.52273 & 99.08578 & 0 \\
\hline & & $\lambda_{g}$ & 0.03457 & 0.73627 & 17.10365 & 0.22597 & 96.30501 & 0 \\
\hline \multirow[t]{2}{*}{2} & 20 & $\lambda_{b}$ & 0.05174 & 1.52248 & 25.93257 & 0.4196 & 149.18541 & 0 \\
\hline & & $\lambda_{g}$ & 0.02187 & 0.16728 & 146.24645 & 0.55973 & 0 & 0 \\
\hline \multirow[t]{2}{*}{3} & 1 & $\lambda_{b}$ & 0.24012 & -0.01907 & $6.09529 \mathrm{E}-4$ & $-8.17819 \mathrm{E}-6$ & $4.83789 \mathrm{E}-8$ & $-1.04568 \mathrm{E}-10$ \\
\hline & & $\lambda_{g}$ & 0.15504 & -0.01238 & $3.96633 \mathrm{E}-4$ & $-5.3329 \mathrm{E}-6$ & $3.16052 \mathrm{E}-8$ & $-6.84288 \mathrm{E}-11$ \\
\hline \multirow[t]{2}{*}{3} & 2 & $\lambda_{b}$ & 0.5452 & 0.00212 & $6.42941 \mathrm{E}-5$ & $-1.46783 \mathrm{E}-7$ & 0 & 0 \\
\hline & & $\lambda_{g}$ & 0.30594 & $-9.58858 \mathrm{E}-4$ & $1.12174 \mathrm{E}-4$ & $-1.04079 \mathrm{E}-6$ & $3.4564 \mathrm{E}-9$ & $-3.91536 \mathrm{E}-12$ \\
\hline
\end{tabular}


Table 2-Continued

\begin{tabular}{|c|c|c|c|c|c|c|c|c|}
\hline Stage & $\operatorname{Mass}\left(M_{\odot}\right)$ & $\lambda$ & $\mathrm{a}$ & $b_{1}$ & $b_{2}$ & $b_{3}$ & $b_{4}$ & $b_{5}$ \\
\hline \multirow[t]{2}{*}{3} & $3^{*}$ & $\lambda_{b}$ & -0.475 & -0.00328 & $1.31101 \mathrm{E}-4$ & $-6.03669 \mathrm{E}-7$ & $8.49549 \mathrm{E}-10$ & 0 \\
\hline & & $\lambda_{g}$ & 0.05434 & 0.0039 & $9.44609 \mathrm{E}-6$ & $-3.87278 \mathrm{E}-8$ & 0 & 0 \\
\hline \multirow[t]{2}{*}{3} & $4^{*}$ & $\lambda_{b}$ & -0.2106 & -0.01574 & $2.01107 \mathrm{E}-4$ & $-6.90334 \mathrm{E}-7$ & 7.92713E-10 & 0 \\
\hline & & $\lambda_{g}$ & 0.36779 & -0.00991 & $1.19411 \mathrm{E}-4$ & $-3.59574 \mathrm{E}-7$ & $3.33957 \mathrm{E}-10$ & 0 \\
\hline \multirow[t]{2}{*}{3} & 5 & $\lambda_{b}$ & -0.12027 & 0.01981 & $-2.27908 \mathrm{E}-4$ & $7.55556 \mathrm{E}-7$ & 0 & 0 \\
\hline & & $\lambda_{g}$ & 0.31252 & -0.00527 & $3.60348 \mathrm{E}-5$ & $-3.22445 \mathrm{E}-8$ & 0 & 0 \\
\hline \multirow[t]{2}{*}{3} & 6 & $\lambda_{b}$ & 0.26578 & 0.00494 & $-7.02203 \mathrm{E}-5$ & $2.25289 \mathrm{E}-7$ & 0 & 0 \\
\hline & & $\lambda_{g}$ & 0.26802 & -0.00248 & $6.45229 \mathrm{E}-6$ & $1.69609 \mathrm{E}-8$ & 0 & 0 \\
\hline \multirow[t]{2}{*}{3} & 7 & $\lambda_{b}$ & 0.8158 & -0.01633 & $1.46552 \mathrm{E}-4$ & $-5.75308 \mathrm{E}-7$ & $8.77711 \mathrm{E}-10$ & 0 \\
\hline & & $\lambda_{g}$ & 0.26883 & -0.00219 & $4.12941 \mathrm{E}-6$ & $1.33138 \mathrm{E}-8$ & 0 & 0 \\
\hline \multirow[t]{2}{*}{3} & 8 & $\lambda_{b}$ & 0.74924 & -0.01233 & $9.55715 \mathrm{E}-5$ & $-3.37117 \mathrm{E}-7$ & $4.67367 \mathrm{E}-10$ & 0 \\
\hline & & $\lambda_{g}$ & 0.25249 & -0.00161 & $8.35478 \mathrm{E}-7$ & $1.25999 \mathrm{E}-8$ & 0 & 0 \\
\hline \multirow[t]{2}{*}{3} & 9 & $\lambda_{b}$ & 0.73147 & -0.01076 & $7.54308 \mathrm{E}-5$ & $-2.4114 \mathrm{E}-7$ & $2.95543 \mathrm{E}-10$ & 0 \\
\hline & & $\lambda_{g}$ & 0.31951 & -0.00392 & $2.31815 \mathrm{E}-5$ & $-6.59418 \mathrm{E}-8$ & $7.99575 \mathrm{E}-11$ & 0 \\
\hline \multirow[t]{2}{*}{3} & 10 & $\lambda_{b}$ & -9.26519 & 0.08064 & $-2.30952 \mathrm{E}-4$ & $2.21986 \mathrm{E}-7$ & 0 & 0 \\
\hline & & $\lambda_{g}$ & 0.81491 & -0.00161 & $-8.13352 \mathrm{E}-6$ & $1.95775 \mathrm{E}-8$ & 0 & 0 \\
\hline \multirow[t]{2}{*}{3} & 12 & $\lambda_{b}$ & -51.15252 & 0.30238 & $-5.95397 \mathrm{E}-4$ & $3.91798 \mathrm{E}-7$ & 0 & 0 \\
\hline & & $\lambda_{g}$ & -13.44 & 0.08141 & $-1.641 \mathrm{E}-4$ & $1.106 \mathrm{E}-7$ & 0 & 0 \\
\hline \multirow[t]{2}{*}{3} & 14 & $\lambda_{b}$ & -140 & 0.7126 & 0.00121 & $6.846 \mathrm{E}-7$ & 0 & 0 \\
\hline & & $\lambda_{g}$ & -44.1964 & 0.22592 & $-3.85124 \mathrm{E}-4$ & $2.19324 \mathrm{E}-7$ & 0 & 0 \\
\hline \multirow[t]{2}{*}{3} & 16 & $\lambda_{b}$ & -358.4 & 1.599 & -0.00238 & $1.178 \mathrm{E}-6$ & 0 & 0 \\
\hline & & $\lambda_{g}$ & -118.13757 & 0.52737 & $-7.8479 \mathrm{E}-4$ & $3.89585 \mathrm{E}-7$ & 0 & 0 \\
\hline \multirow[t]{2}{*}{3} & 20 & $\lambda_{b}$ & -436.00777 & 1.41375 & -0.00153 & $5.47573 \mathrm{E}-7$ & 0 & 0 \\
\hline & & $\lambda_{g}$ & -144.53456 & 0.46579 & $-4.99197 \mathrm{E}-4$ & $1.78027 \mathrm{E}-7$ & 0 & 0 \\
\hline
\end{tabular}

${ }^{*}$ Rows with the $\operatorname{symbol} *$ use $\log \lambda_{b}$ instead of $\lambda_{b}$ as $y$ variables. 